\title{
Rol de la diplomacia pública en la VI Cumbre de las Américas ${ }^{1}$
}

DeISSY VIVIANA DURÁN RODRÍGUEZ ${ }^{2,}$ *

\section{Resumen}

La VI Cumbre de las Américas fue realizada en medio de un contexto singular del sistema internacional, en el cual, el debate en torno a la inserción de Cuba en eventos multilaterales americanos y la discusión sobre la soberanía de las Islas Malvinas tomaron gran relevancia debido a los eventos acontecidos previamente. La discusión sobre estos temas impidió que la cumbre concluyera con una declaración final, por lo que muchos abrieron un debate en torno a la pertinencia de la realización de estos eventos, ya que, de ellos se esperan como resultado consensos, pero una vez más se demostró que es muy difícil llegar a ellos en estos escenarios. Aunque algunos han señalado que hubo un mal manejo diplomático colombiano para desarrollar la cumbre, se debe entender que en este tipo de eventos en donde la diplomacia es pública, cada presidente asistente busca tomar actitudes acordes a lo esperado por la opinión pública de su país, lo que constituye, el verdadero problema que tienen estos escenarios para que se dé un consenso.

Palabras clave: Cumbre de las Américas, diplomacia pública, Cuba, Islas Malvinas, consenso.

Clasificación JEL: F02, F15, F43, F49.

\footnotetext{
${ }^{1}$ Artículo de investigación.

2 Profesional en Relaciones Internacionales y Estudios Políticos de la Universidad Militar Nueva Granada. *dviviana@gmail.com.
}

Fecha de recepción: 4 de septiembre de 2017.

Fecha de aceptación: 3 de diciembre de 2017.

Para citar este artículo: Durán, D. (2018). Rol de la diplomacia pública en la VI Cumbre de las Américas. Perspectivas en inteligencia, 10(19): 193-205. 


\section{Abstract}

The VI Summit of the Americas was held in the midst of a unique context of the international system, in which, the debate about the insertion of Cuba in American multilateral events and the discussion on the sovereignty of the Falkland Islands took on great relevance due to the events previously occurred. The discussion on these issues prevented the summit from concluding with a final statement, so many opened a debate about the relevance of the realization of these events, since they are expected as a result of consensus, but once again He showed that it is very difficult to reach them in these scenarios. Although some have indicated that there was a Colombian diplomatic mismanagement to develop the summit, it should be understood that in this type of events where diplomacy is public, each assistant president seeks to take attitudes according to what is expected by the public opinion of his country, what constitutes, the real problem that these scenarios have so that a consensus is given.

Keywords: Summit of the Americas, public diplomacy, Cuba, Malvinas Islands, consensus.

JEL classification: F02, F15, F43, F49. 


\section{Introducción}

Las cumbres diplomáticas se realizan con el propósito de discutir sobre temas de interés común interestatal, en donde, se manifiestan demandas, se exponen las posturas de cada estado participante y se debaten asuntos específicos, manifestándose, los puntos en donde hay acuerdos y desacuerdos, con el fin, de llegar a aprobaciones, sobre cómo enfrentar situaciones que tienen un valor importante para los estados que asisten a tales escenarios.

En Cartagena de Indias (Colombia), se dio lugar a la realización de la VI versión de la Cumbre de las Américas, en la cual, se habló de las situaciones que se estaban presentando en el sistema internacional, se debatieron temas catalogados como espinosos, que dificultaron alcanzar un consenso entre los participantes, lo cual se vio reflejado en la ausencia de una declaración final.

Tras esta cumbre, varios sectores de la opinión pública han comentado que al ser difícil alcanzar un consenso -lo cual constituye uno de los objetivos de estos eventos- se debería reevaluar la pertinencia de continuar su realización, pues, aunque se discuten varios temas, no se llega a un acuerdo claro, en donde, se definan líneas de acción a ejecutar y se diseñen programas concretos que vayan más allá de los discursos.

Es así como la realización de la VI Cumbre de las Américas pone en discusión la pertinencia de la organización de eventos, en donde la diplomacia toma un carácter público, aumentando la dificultad que tiene la diplomacia multilateral para consolidar un consenso.

Sin embargo, para poder determinar la valía de estos eventos hay varios factores que se deben tener en cuenta, ya que, como se ha mencionado, la diplomacia multilateral reúne diferentes Estados con diversidad de intereses particulares sobre materias que afectan a otras naciones, lo que dificulta el conseguir un consenso, debido a razones como: Las diferencias ideológicas, la necesidad de mostrar el fortalecimiento de un Estado en el Sistema Internacional o la búsqueda del beneplácito de los ciudadanos de cada país representado -aspecto sobresaliente en contextos de diplomacia pública-.

Dicha discusión temática en escenarios multilaterales, conlleva a que no resulte en programas de acción, pero sí se permita el diálogo sobre temas vitales y se dé lugar a que el público conozca lo que se está organizando en las altas esferas del poder. 
En el contexto del continente americano, se debe tener en cuenta que los países del hemisferio, han presentado tradicionalmente una evidente falencia de autonomía frente a Estados Unidos, quien, ha direccionado las políticas y líneas de acción en todo tipo de ámbitos, sin embargo, en los últimos años se ha mostrado una mayor independencia e incluso una tendencia a crear parámetros contrarios a los establecidos por el país norteamericano.

Durante la realización de la VI versión de la Cumbre de las Américas, se evidenció la división en las perspectivas que hay frente a temas cruciales para el continente como Cuba y la soberanía de las Islas Malvinas, con respecto a esto, hay una postura casi generalizada en el hemisferio, excepto, por Estados Unidos y Canadá, países por los cuales no se llegó a un acuerdo.

Es importante entender, que fue por la iniciativa del ex presidente estadounidense Bill Clinton, que se empezaron a realizar las Cumbres de las Américas, y que muchos expertos han señalado que los consensos que se lograban en los anteriores eventos se debían a la imposición de la visión del país estadounidense.

Aunque en la VI Cumbre de las Américas, los temas planteados en la agenda estaban relacionados con los debates en torno a problemáticas como la pobreza, la equidad y el crecimiento, sin lugar a duda, importantes para América. Por otra parte, fueron Cuba y Malvinas los que impidieron llegar a un consenso, lo que demuestra, la gran relevancia que aún ocupan estos asuntos surgidos en el contexto de la guerra fría.

\section{Cumbres: diplomacia pública}

Es necesario tener en cuenta, que las Cumbres son un espacio abierto a los medios de comunicación, por lo que, el público en general puede estar al tanto de lo que acontece con cada uno de los mandatarios. Lo cual, permite entender más fácilmente la actitud y postura que mantuvieron los diferentes presidentes que formaron parte del debate de los temas vigentes durante la Cumbre, porque estarían siendo analizados por la opinión pública de sus respectivos países, en asuntos que pueden tener gran influencia en la política interior y qué pueden representar para los actuales presidentes.

Esta situación, fue la que enfrentaron principalmente el ex presidente Obama y la ex presidenta Kirchner, ya que, los asuntos de Cuba y Malvinas; Por un lado, Obama se encontraba, en campaña para su reelección en Estados Unidos, 
por lo que debía manejar públicamente una postura acorde a los parámetros tradicionales de la política norteamericana, como lo es en el caso de Cuba, exigir la implementación de un régimen democrático y el respeto a los derechos humanos como condiciones innegociables para que la isla pueda participar en los escenarios multilaterales hemisféricos, bajo la perspectiva política de los norteamericanos.

Frente al caso de la presidenta de Argentina, el tema de las islas Malvinas es muy sensible para sus nacionales, ya que, este país en este tema ha sido abordado para la desviación de otros problemas internos, usando como pretexto la reclamación de las islas como parte de la soberanía argentina. Cristina Kirchner se retiró de la Cumbre horas antes de su clausura, tras conocerse que no se había llegado a un consenso en el caso de las Malvinas, esa reacción, puede ser percibida con beneplácito por sus nacionales.

\section{Invitar a Cuba a las Cumbres de América}

El tema de permitir que Cuba participe en la Cumbre de las Américas, en la VI versión realizada en la ciudad de Cartagena, el debate frente a esta situación tuvo más fuerza, debido al cambio de postura de los diferentes países americanos frente a la exclusión de la isla en los procesos multilaterales del continente.

El triunfo de la revolución cubana en enero de 1959, enmarcado en el escenario bipolar de la guerra fría, representó que la presencia socialista se encontraba cercana al territorio estadounidense, posición ideológica que fue tangible cuando el gobierno cubano decidió expropiar las empresas norteamericanas que se encontraban en la isla.

La afinidad de un país del continente americano con la tendencia socialista, causó malestar entre los países del hemisferio, cuyos gobiernos, manifestaron públicamente su rechazo al comunismo (Declaración de San José, 1960, s.p.) contra la intervención soviética en el continente americano.

La influencia de los soviéticos en el continente americano, pese a que en su gran mayoría rechazaba las tendencias comunistas, se hizo pública en enero de 1962 en Uruguay, durante la VIII reunión de consulta de ministros de relaciones exteriores, en la cual, Estados Unidos buscó ejercer presión para que Cuba cambiará su régimen político.

Aunque, eran varios los países americanos inconformes con el acercamiento de Cuba con los soviéticos, no había una postura común sobre las medidas 
que se debían aplicar al respecto. La presión norteamericana consiguió que se adoptará finalmente la resolución denominada "Ofensiva del comunismo en América", en la que se declaró que el sistema americano era incompatible con los principios comunistas, expulsando de esta manera a Cuba de la Organización de los Estados Americanos (OEA).

A pesar, de la expulsión de la isla de los procesos multilaterales americanos, Cuba supo manejar la situación a través de relaciones bilaterales, impidiendo aislarse del resto del continente. Esto permitió que, en 1987, durante la primera Cumbre del Grupo de Río, varios jefes de Estado estuvieran de acuerdo en reincorporar a Cuba a la OEA, posición que perduró en las siguientes reuniones, bajo la premisa de ajuste a ciertos cambios que se debían dar en la isla. Al ser Cuba un satélite soviético, Estados Unidos rechazó constantemente esta posición, a menos que se diera un cambio radical en la posición comunista del país castrista, lo que desde entonces ha dificultado el consenso.

El gran debate frente a permitir que Cuba sea de nuevo parte de la OEA, está sustentado en el hecho que uno de los principios fundamentales de la organización establece que sus integrantes sean países democráticos.

La posición frente a Cuba, ha venido cambiando tras 50 años de bloqueo, con el que se buscaba presionar a la isla para que cambiara su régimen al democrático, acciones que no han dado el resultado esperado, pues el país sigue inmerso en el sistema socialista con serios problemas económicos que han perjudicado la calidad de vida de la población cubana.

Fue por esto, que el 3 de junio de 2009, durante la XXXIX Asamblea General de la OEA en Honduras, se derogó la suspensión de 1962, quedando sin efectos la declaración que había excluido a la isla, permitiéndose así su reincorporación en la OEA, siempre y cuando acepte cumplir los principios de la Carta de la Organización, respeto a la democracia y al respeto de los derechos humanos.

La Primera Cumbre de las Américas, se realizó en 1994 en Miami, Florida, la idea fue del presidente norteamericano Bill Clinton, el cual, pretendía impulsar la integración del hemisferio. Desde el origen de estas Cumbres, se impartió que a este espacio solo podían asistir los países democráticos, razón por la cual Cuba nunca ha asistido a estos eventos.

En el actual contexto, en el que se ha abolido el precepto histórico que impedía la participación de la isla en los sistemas multilaterales americanos, fue amplio el debate referente a la invitación para que Cuba participará en la VI Cumbre de las Américas cuya sede sería Colombia. 
Allí fue, el presidente ecuatoriano Rafael Correa, quien en nombre de la Alianza Bolivariana de los pueblos de América (ALBA) exigió que Cuba fuera invitada, aun cuando los demás países miembros de esta organización -Venezuela, Bolivia, Nicaragua, Ecuador, San Vicente y las Granadinas, Dominica, y Antigua y Barbuda-, no asistieron a la reunión.

Aunquela diplomacia colombiana se movilizó y buscó el diálogo con los países del ALBA para impedir que esto sucediera, finalmente sólo el presidente ecuatoriano se mantuvo en la decisión, y San Vicente y las Granadinas, Dominica y Antigua y Barbuda, fueron los únicos países miembros del ALBA que permanecieron en el evento. De los demás, el único presidente que se presentó en la cumbre fue el boliviano Evo Morales, el cual se retiró sin asistir a la reunión privada que llevaron a cabo los presidentes de los Estados americanos.

Tras la Cumbre, estados como Brasil, Argentina y los integrantes del ALBA, ratificaron que, para la próxima cumbre, que tendrá lugar en Panamá, no asistirán a menos que Cuba pueda acudir al evento, frente a lo cual Estados Unidos ratificó su postura referente a que Cuba aún no cumple con los requisitos para poder asistir a la Cumbre de las Américas.

Respecto a lo anterior, el secretario general de la OEA, José Miguel Insulza mencionó que Cuba no constituye una problemática regional, sino un tema específico de Estados Unidos, por lo que no existe razón para que Cuba no pueda asistir a eventos multilaterales americanos.

\section{El tema de Malvinas}

Las islas Malvinas, como son llamadas por los argentinos o Falkland por los británicos, ubicadas a 480 kilómetros de la costa patagónica, han sido históricamente un importante referente de debate sobre soberanía.

Este archipiélago, conformado por más de 100 islotes y dos islas principales: Gran Malvina y Soledad, fue disputado en el siglo XVIII por España, Francia y Gran Bretaña; aunque, finalmente España tuvo control sobre este territorio, Gran Bretaña las había considerado parte de su soberanía en 1765.

Tras la independencia argentina de España, el nuevo país americano declaró en 1820 las islas como parte de su territorio, fue entonces cuando en 1833 Gran Bretaña las invadió reclamando su soberanía, declarándolas colonia en 1892; desde entonces, estas islas han estado bajo el dominio británico. 
En 1982, estando Argentina bajo la presidencia de Leopoldo Fortunato Galtieri, en un contexto de descontento ciudadano, derivado de la situación económica y política del país, llevó a que en el mes de marzo se organizaron varias manifestaciones como la movilización que se dio en la Plaza de Mayo, el gobierno de Galtieri, decidió dar paso a la Operación Rosario, con la que se pretendía retomar el dominio argentino sobre las islas Malvinas, señalado este acontecimiento por muchos expertos como una forma en la que el gobierno pretendía desviar la atención que había sobre los problemas internos hacia un tema que era muy importante y significativo para los argentinos, recuperar las Islas Malvinas.

Es así, como el 2 de abril de 1982 desembarcan en Puerto Stanley, capital de las islas, aproximadamente 5 mil efectivos argentinos, el gobierno británico había dejado a cuarenta y nueve marines la misión de la protección del archipiélago, los marines y el gobernador que se encontraban en la isla fueron capturados por las tropas argentinas y llevados a Montevideo.

La entonces jefe de Gobierno británico Margaret Thatcher, movilizó su diplomacia logrando que la ONU considerará a Argentina, como un Estado Agresor y obteniendo de igual forma el respaldo de Estados Unidos y la Comunidad Económica Europea, quienes incluso impusieron sanciones económicas contra el Estado suramericano. El despliegue de equipamiento militar por parte de ambos bandos, permitió un combate que duró hasta el 14 de junio de 1982 cuando las tropas argentinas se rindieron.

Con la victoria de Gran Bretaña, los habitantes de las islas obtuvieron la ciudadanía británica y el gobierno inglés ha fortalecido desde entonces, la custodia sobre el archipiélago, por lo que hay 1500 soldados, buques de guerra, aviones y helicópteros ingleses en las islas, Sin embargo, Argentina nunca ha dejado de reconocerlas como parte de su soberanía, por lo que la disputa continúa, agravándose por la potencial posibilidad de que las aguas de las islas sean ricas en gas y petróleo.

En el 2012 se cumplió el aniversario número treinta del enfrentamiento, y la expresidenta argentina Cristina Kirchner ha lanzado una dinámica ofensiva que buscaba una negociación con Gran Bretaña, para recuperar la soberanía delas islas, por lo que la Cumbre de las Américas fue vista por Kirchner como un escenario propicio para discutir el tema, con el fin, de obtener el respaldo de los países americanos a la causa argentina, refiriéndose, al respecto, el canciller argentino Héctor Timerman, en la reunión sostenida antes de la Cumbre mencionando que "la agresión colonial contra un país es la agresión colonial a todos". 
Sin embargo, el único discurso de la Cumbre transmitido, fue el inaugural, realizado por el expresidente Juan Manuel Santos, el cual, no hizo referencia al tema de las Malvinas, cosa que la expresidenta de Argentina le reclamó al mandatario colombiano, como él mismo lo reconoció en la conferencia de prensa.

Aunque muchos países del continente apoyan la postura que las Malvinas, deben ser argentinas o por lo menos debe haber una negociación con Gran Bretaña sobre el asunto, y se refirieron al respecto denominándolo un asunto colonial, Estados Unidos y Canadá fueron los países por los que no hubo consenso sobre este punto.

Por un lado, Estados Unidos como ya se mencionó, aunque apoyó durante el conflicto a Gran Bretaña, en la Cumbre, manifestó que mantendría una postura neutra frente al asunto, aunque en la rueda de prensa final Obama se refirió al respecto diciendo "anticipamos positivamente la posibilidad que ellos mantengan un diálogo positivo”, es claro, que el país norteamericano es un aliado natural de Gran Bretaña. Por otro lado, en lo que respecta a Canadá, no hay que olvidar que el país forma parte del Commonwealth, organización dirigida por la reina Isabel II y que, por lo tanto, respalda los intereses británicos.

El ex presidente Andrés Pastrana, en la entrevista hecha por caracol radio sobre la Cumbre, declaró que a Colombia le había ido mal en materia de diplomacia, entre otras cosas porque el hecho que el debate frente al tema de las Malvinas se había venido acentuando en las semanas previas a la realización del evento, hacía que Colombia tuviera una posición diplomática frente al tema o que hubiera hecho una declaración al respecto, y resaltó que eso era lo que estaba esperando Argentina, no tanto que América tomará una posición definitiva sobre este asunto.

El profesor de derecho diplomático y consular de la Universidad Militar Nueva Granada, Mauricio Pryor, señala por el contrario que Colombia, al no haber hecho una declaración pública sobre el asunto, fue prudente y asumió una postura inteligente, ya que, la disputa por las Islas Malvinas tiene similitudes con la existente entre Colombia y Nicaragua por los límites marítimos en el Caribe, controversia que envuelve la soberanía sobre los componentes insulares del archipiélago de San Andrés, además cerca de 50.000 km2 en el océano Atlántico, importante, no sólo por lo que representa este océano en lo que respecta al transporte comercial, sino, por su riqueza en recursos naturales y minerales, incluidos yacimientos de petróleo. 
Esta disputa, que mantiene Colombia con Nicaragua se está adelantando en los tribunales de la Corte Internacional de Justicia en un juicio que fue retomado en el mes de abril de 2012, pocos días después de la realización de la VI Cumbre de las Américas.

Para Colombia, que ha hecho presencia histórica en la zona y que ha considerado al Tratado de 1928 o Tratado Esguerra - Barcenas- ratificado por el Protocolo de 1930, como el sustento para reclamar este territorio como parte de la soberanía colombiana, no es conveniente mostrar una posición respecto al caso argentino, ya que, Nicaragua reclama que los límites establecidos no corresponden con lo establecido por el derecho del mar.

\section{Ausencia de una declaración final}

Uno de los aspectos más criticados de la VI Cumbre de las Américas es que en esta oportunidad no hubo una declaración final, lo que fue catalogado por muchos como un fracaso para la diplomacia colombiana ya que este tipo de escenarios son para lograr consensos entre los países asistentes.

No obstante, el presidente de Colombia declaró que lo que había que resaltar era el hecho de haber tocado temas controversiales, delicados, que usualmente presentan discrepancias significativas, sobre los cuales era muy difícil que los países asistentes se pusieran de acuerdo y que esto era bueno en la medida en que no se dio una declaración final sobre asuntos que normalmente se manejan fácilmente pero se resaltaba a Colombia como el escenario en el que se empezaron a debatir temas cruciales para América, sobre los cuales era visible que no se daría una conclusión final pero si se abría el espacio para su debate.

Lo que sobresale de los debates frente a temas como Cuba y Malvinas es que la concepción sobre estos asuntos es muy similar entre los países de América, excepto por Estados Unidos y Canadá. De hecho, se expresó que no hubo declaración debido a que Estados Unidos vetó el artículo que mencionaba que Cuba podría asistir a la próxima Cumbre, y que esto fue lo que anuló la declaración final ya que la posición norteamericana impedía el consenso. La existencia de una posición común entre los demás Estados americanos también es muestra de una mayor independencia y autonomía frente a Estados Unidos.

Hay expertos que señalan que hubo falencias diplomáticas en la medida en que temas como el de Cuba y las Malvinas pudieron haberse trabajado más 
contundentemente antes de la realización de la Cumbre, de tal forma que en el transcurso del evento no hubieran sido tocados estos temas, los cuales no hacían parte de la agenda oficial preestablecida.

\section{Conclusiones}

El proceso que se ha venido dando durante los últimos años en el continente americano, en el cual la mayoría de los Estados se han mostrado de acuerdo con la reintegración de Cuba en los escenarios multilaterales, fue muy importante durante la realización de la VI Cumbre de las Américas, en cuanto varios de los países han expresado que dejaran de asistir a estos eventos si la isla no es invitada, lo que muestra una mayor independencia y autonomía frente a las posiciones establecidas por Estados Unidos.

La no consolidación de una declaración respecto a la disputa entre Argentina y Gran Bretaña por las islas Malvinas fue muestra de prudencia del Gobierno Colombiano, ya que Colombia enfrenta actualmente una situación similar por el juicio que se adelanta en la Haya respecto a los límites marítimos con Nicaragua.

Al ser las Cumbres escenarios de debate interestatal expuestos al público, los mandatarios participantes tratan de manejar posturas que sean aprobadas por los habitantes de sus respectivos países.

La falta de una declaración final en la VI Cumbre de las Américas, muestra la división de posturas entre Estados Unidos y Canadá, por un lado, y el resto del continente por el otro, ya que en temas como Cuba y Malvinas hay posiciones afines entre los países americanos excepto por estos Estados. 


\section{Referencias}

1. Aguilera, P. G. (2012). Apuntes de una larga y complicada relación En: Nueva Sociedad No. 223. Recuperado de: http://www.nuso.org/upload/articulos/3628_1.pdf

2. Balinotti, N. (2012). Mantendrá Gran Bretaña la fuerza militar en Malvinas. Recuperado de: http://www.lanacion.com.ar/1462066-mantendra-gran-bretana-la-fuerza-militar-enmalvinas

3. Caracol Radio (2012). Me sorprende que Pastrana califique la Cumbre de las Américas como un fracaso diplomático: Canciller. Recuperado de: http://www.caracol.com.co/ noticias/actualidad/me-sorprende-que-pastrana-califique-la-cumbre-de-las-americascomo-un-fracaso-diplomatico-canciller/20120420/nota/1673332.aspx

4. Caracol Radio (2012). Nos fue mal en materia diplomática en la Cumbre de las Américas: Andrés Pastrana. Recuperado de: http://www.caracol.com.co/noticias/actualidad/nos-fuemal-en-materia-diplomatica-en-la-cumbre-de-las-americas-andres-pastrana/20120418/ oir/1671924.aspx

5. CNN en español. (2012). Las claves del conflicto entre Gran Bretaña y Argentina por las Malvinas. Recuperado de: http://cnnespanol.cnn.com/2012/02/08/las-claves-delconflicto-entre-gran-bretana-y-argentina-por-las-malvinas/

6. Cumbre de las Américas (s.f). Recuperado de: http://www.summit-americas.org/defaults. htm (2012, 23 abril)

7. El Liberal (2012). Colombia defiende su histórica soberanía en islas reclamadas por Nicaragua. Recuperado de: http://www.elliberal.com.co/liberal/actualidad/ mundo/110353-colombia-defiende-su-historica-soberania-en-islas-reclamadas-pornicaragua

8. El Mundo (2012). Alba pide presencia de Cuba en Cumbre de las Américas. Recuperado de: http://www.elmundo.com/portal/noticias/nacional/alba_pide_presencia_de_cuba_ en_cumbre_de_las_americas.php

9. El Mundo (2009). La OEA hace caso omiso a EEUU y acuerda readmitir a Cuba sin condiciones. Recuperado de: http://www.elmundo.es/elmundo/2009/06/03/ internacional/1244054562.html

10. El Nuevo Heraldo (2012). Cumbre de las Américas sin consenso sobre Malvinas. Recuperado de: http://www.elnuevoherald.com/2012/04/15/1179272/cumbre-de-lasamericas-sin-consenso.html

11. El País (2012). Cuba no cumple requisitos para asistir a Cumbre, dice Estados Unidos. Recuperado de: http://www.elpais.com.co/elpais/internacional/noticias/eeuu-cubacumple-requisitos-para-asistir-cumbre

12. FLACSO (2004). Gobernabilidad, seguridad y diplomacia de cumbres. Recuperado de: http:// www.flacso.org/fileadmin/usuarios/David/11.pdf.

13. Gómez, M.S. (2012). No hay razón para excluir a Cuba de cumbres de las Américas: OEA. Recuperado de: http://www.eltiempo.com/mundo/latinoamerica/ARTICULO-WEBNEW_NOTA_INTERIOR-11644601.html

14. Hernández, M. (2012). Cubay Las Malvinas impiden el consenso en la VI Cumbre de las Américas. Recuperado de: http://www.elmundo.es/america/2012/04/15/colombia/1334506743. html

15. La Nación (2012). Argentina pidió apoyo por las Malvinas a los países reunidos en Cartagena. Recuperado de: http://www.lanacion.com.ar/1464705-argentina-pidio-apoyo-por-lasmalvinas-a-los-paises-reunidos-en-cartagena (2012, 25 abril) 
16. La Nación (2012). La Presidenta exige diálogo y promete respeto a los isleños. Recuperado de: http://www.lanacion.com.ar/1461820-cont-cristina-kirchner-exige-dialogo-a-londres

17. La Nación (2012). Partió rumbo a Malvinas el destructor británico que desató la polémica. Recuperado de: http://www.lanacion.com.ar/1462170-partio-rumbo-a-malvinas-eldestructor-britanico-que-desato-la-polemica

18. Pearson, F. y Rochester, J. (2000). Relaciones Internacionales, Situación global en el siglo XXI. $4^{\mathrm{a}}$ ed., Bogotá: McGraw-Hill Interamericana, S. A., 229-262.

19. Revista Semana (2007). En Nicaragua celebran lo que consideran un enorme triunfo: "Ganamos pelea por frontera marítima". Recuperado de: http://www.semana.com/on-line/nicaraguacelebran-consideran-enorme-triunfo-ganamos-pelea-frontera-maritima/108314-3.aspx

20. Terra (2012). Chávez buscará que Correa asista a la Cumbre, dice Holguín. Recuperado de: http://noticias.terra.com.co/nacional/chavez-buscara-que-correa-asista-a-la-cumbredice-holguin,9a2a9581f92f5310VgnVCM20000099f154d0RCRD.html

21. Vega, W (2012). Evo Morales no irá a retiro de presidentes en Cumbre de las Américas. Recuperado de: http://www.eltiempo.com/mundo/latinoamerica/ARTICULO-WEBNEW_NOTA_INTERIOR-11572421.html

22. Yahoo Noticias (2012). Se declara Obama neutral en diferendo por Malvinas. Recuperado de: http://mx.noticias.yahoo.com/declara-obama-neutral-diferendo-malvinas-234700520. html. 